\title{
Analysis of Beam-Beam Kink Instability in a Linac-Ring Electron-Ion Collider
}

\author{
R. Li, B. C. Yunn, Jefferson Lab, Newport News, USA * \\ V. Lebedev, FNAL, Batavia, IL 60510, USA \\ J. J. Bisognano, SRC, Univ. of Wisconsin-Madison, USA
}

\begin{abstract}
A linac-ring collision scheme was considered in recent proposals of electron-gold colliders (eRHIC) and polarized-electron light-ion colliders (EPIC). The advantages of using an energy-recovered linac for the electron beam is that it avoids the limitation of beam-beam tune shift inherent in a storage ring, pertains good beam quality and easy manipulation of polarization. However, the interaction of the ion beam in the storage ring with the electron beam from the linac acts analogously to a transverse impedance, and can induce unstable behavior of the ion beam similar to the strong head-tail instability. In this paper, this beam-beam kink instability with head-tail effect is analysed using the linearized Vlasov equation, and the threshold of transverse mode coupling instability is obtained.
\end{abstract}

\section{INTRODUCTION}

A linac-on-ring collision scheme, where a linac beam collides with a storage ring beam, was earlier proposed [1] based on the idea that in such colliders the charge density for the storage ring beam is no longer limited by the beam-beam tune shift of the linac beam, hence a higher luminosity can be achieved. The interest of linac-ring collision scheme is renewed in recent proposals of electron-gold colliders (eRHIC) and polarized-electron light-ion collider$s$ (EPIC)[2]. Our previous study of the beam-beam effects in a linac-on-ring B factory revealed that coherent beambeam head-tail dipole instability could set strong limit to luminosity in this collision scheme. This dipole instability was first observed in our strong-strong beam-beam simulation [3] in a linac-ring B factory, and later confirmed by our analysis of the initial value problem of the system in the linear growth regime when synchrotron oscillation is turned off [4]. Recent simulation of linac-ring beam-beam effects by Perevedentsev and Valishev [5] further demonstrated the head-tail instability due to the betatron phase advance over the beam-beam interaction region, as well as the strong head-tail instability due to the linac-ring beambeam interaction. In this paper, the stability of a stored ion bunch, under collision with a short electron bunch, was studied using the linearized Vlasov equation. It is shown that the linear beam-beam kick between electron and ion bunches acts like a broad-band transverse impedance to the dipole moment of the ion slices. Using conventional treatment of transverse mode coupling instability, we obtained threshold for the strong head-tail beam-beam kink insta-

\footnotetext{
* This work was supported by the U.S. DOE Contract No. DE-AC0584ER40150.
}

bility. We also find that due to the localized beam-beam kick, small growth rate exists before reaching the transverse mode coupling threshold. It should be emphasized that the betatron phase advance through the interaction region, often called hourglass effect, is not yet included in our current study. Some initial estimations [6] show that this effect could cause serious head-tail instability before the strong head-tail instability takes place. Further analytical study including the hourglass and chromaticity effects, as well as numerical study using the strong-strong beambeam simulation with actual nonlinear beam-beam interaction force, is currently underway.

\section{VLASOV EQUATION ANALYSIS}

For this analysis, we consider the dynamics of vertical dipole moments of an ion bunch $\left(\sigma_{x+} \gg \sigma_{y+}\right)$ in a storage ring colliding with an electron bunch $\left(\sigma_{x-} \gg \sigma_{y-}\right)$ from a linac. The ion bunch with bunch length $l_{+}$is much longer than the electron bunch, thus the electron bunch is described by a $\delta$-like slice. For simplicily of analysis, the ion bunch is chosen to have uniform longitudinal charge density, and the rms bunch sizes $\sigma_{x+}$ and $\sigma_{y+}$ are uniform along the bunch. Note that here we assume $\beta_{y+}^{*} \gg l_{+}$at IP, so the hourglass effect on synchrobetatron coupling is not included.

Let $z$ be the longitudinal coordinate in the ion bunch, $-l_{+} / 2 \leq z \leq l_{+} / 2$, and consider the first interaction which occurs during $-l_{+} / 4 \leq s \equiv c t \leq l_{+} / 4$. Due to linear beam-beam kick in terms of the focusing length $f_{+}$, with $f_{+}^{-1}=2 N_{-} r_{i} / \gamma_{+} \sigma_{y-}\left(\sigma_{x-}+\sigma_{y-}\right)$ and $r_{i}=$ $q_{i} e^{2} / m_{i} c^{2}$, the motion of the vertical coordinate $y_{+}(z, s)$ of an ion particle is given by

$$
\frac{d y_{+}}{d s}=u_{y+}, \quad \frac{d u_{y+}}{d s}=-\delta\left(s-\frac{z}{2}\right) \frac{y_{+}-\bar{y}_{-}(z, s)}{f_{+}},
$$

where $\bar{y}_{-}(z, s)$ is the verticle offset of the electron slice at the encounter with the ion slice at $z$. Similarly, if we simplify $y_{ \pm}(z, s)$ for $s=z / 2$ as $y_{ \pm}(z)$, we have for the ion slice at $z$

$$
\frac{d \bar{y}_{-}}{d s}=\bar{u}_{y-}, \quad \frac{d \bar{u}_{y-}}{d s}=-\lambda_{z+} \frac{\bar{y}_{--}-\bar{y}_{+}(z)}{f_{-}} .
$$

with $f_{-}^{-1}=2 N_{+} r_{e} / \gamma_{-} \sigma_{y+}\left(\sigma_{x+}+\sigma_{y+}\right)$. Here the longitudinal ion density is constant: $\lambda_{z+}=1 / l_{+}$, hence the linear beam-beam kick strength on $e^{-}$from all longitudinal ion slices is uniform. Let $k_{-}^{2}=1 / l_{+} f_{-}$, and assuming zero offset and deflection of the electron slice from the linac, we get from Laplace transform of Eq. (2)

$$
\bar{y}_{-}\left(z_{+}\right)=\frac{k_{-}}{2} \int_{-l_{+} / 2}^{z_{+}} \sin \frac{k_{-}}{2}\left(z_{+}-z_{+}^{\prime}\right) \bar{y}_{+}\left(z_{+}^{\prime}\right) d z_{+}^{\prime} .
$$


Applying Eq. (3) to Eq. (1), we obtain the general description of the ion vertical motion in the whole ring

$$
\begin{aligned}
& \frac{d y_{+}}{d s}=u_{y+}, \quad \frac{d u_{y+}}{d s}+k_{\beta}^{2} y_{+}=\frac{F_{y}(z, s)}{E} \\
& \frac{F_{y}(z, s)}{E}=-\sum_{n=0}^{\infty} \delta\left(s-\frac{z}{2}-n C\right) \frac{y_{+}-\bar{y}_{-}}{f_{+}},
\end{aligned}
$$

with $k_{\beta}=\omega_{\beta} / c$ accounts for the sinusoidal betatron oscillation, $C$ the circumference of the ring, and $n$ the number of collisions. Note here the electron bunch disruption parameter $D_{-}$and the ion bunch beam-beam tune shift $\xi_{+}$are related to $f_{-}, f_{+}$and betatron function $\beta_{y+}^{*}$ at IP by

$$
D_{-}=\sigma_{z+} / f_{-}, \quad \xi_{+}=\beta_{y+}^{*} / 4 \pi f_{+} .
$$

With Eqs. (3)-(5), we can now focus on the stability of the stored ion beam following the standard treatment of transverse collective instability [7]. Let $y$ be the vertical coordinate of an ion particle, $u_{y}=d y / d s, z$ the distance from the synchronous particle and $\delta$ the relative energy deviation from designed energy. The synchrotron motion is given by $d z / d s=-\eta \delta$ and $d \delta / d s=\left(k_{s}^{2} / \eta\right) z+$ $(y / E) \partial F_{y}(z, s) / \partial z \approx\left(k_{s}^{2} / \eta\right) z$ for $k_{s}=\omega_{s} / c$. Using the action-angle transform $y=q \cos \theta, u_{y}=-k_{\beta} q \sin \theta$ and $z=r \cos \phi, \eta \delta / k_{s}=r \sin \phi$, we get the Vlasov equation for the phase space distribution function with the new variables $f(q, \theta, r, \phi, s)$

$$
\frac{\partial f}{\partial s}+k_{\beta} \frac{\partial f}{\partial \theta}+k_{s} \frac{\partial f}{\partial \phi}+\frac{F_{y}(z, s)}{E} \frac{\partial f}{\partial u_{y}}=0 .
$$

Furthermore, we write $f(q, \theta, r, \phi, s)$ as

$$
f(q, \theta, r, \phi, s)=f_{0}(q) g_{0}(r)+f_{1}(q, \theta, r, \phi, s)
$$

where $f_{0}(q)$ describes the equilibrium betatron phase space distribution and $g_{0}(r)$ the equilibrium longitudinal phase space distribution, and $f_{1}(q, \theta, r, \phi, s)$ stands for the perturbation from the equilibrium distribution. For uniform longitudinal distribution, we have for $\hat{z}=l_{+} / 2$

$$
g_{0}(r)=\frac{N_{+} \eta}{2 \pi k_{s} \hat{z}} \frac{1}{\sqrt{\hat{z}^{2}-r^{2}}} \quad(r \leq \hat{z}) .
$$

Consider only the dipole motion, we have

$f_{1}(q, \theta, r, \phi, s)=-\frac{\partial f_{0}}{\partial q}\left[e^{i \theta} g_{+}(r, \phi, s)+e^{-i \theta} g_{-}(r, \phi, s)\right]$,

and the dipole offset of the ion slice at $z$ is

$$
\bar{y}(z, s)=\frac{l_{+}}{N_{+}} \int_{-\infty}^{\infty} d \delta\left[g_{+}(r, \phi, s)+g_{-}(r, \phi, s)\right] .
$$

Combining terms with $e^{ \pm i \theta}$, and expending $g_{ \pm}(r, \phi, s)$ in terms of longitudinal modes,

$$
g_{ \pm}(r, \phi, s)=\sum_{l=-\infty}^{\infty} R_{l}^{ \pm}(r, s) e^{i l \phi}
$$

we get from Eq. (7) the linearized equation for $R_{l}^{ \pm}(r, s)$ :

$$
\begin{aligned}
& \sum_{l=-\infty}^{\infty}\left[\frac{\partial R_{l}^{ \pm}}{\partial s}\right.\left.+i\left( \pm k_{\beta}+l k_{s}\right) R_{l}^{ \pm}\right] e^{i l \phi} \\
& \mp \frac{i}{2 k_{\beta}} g_{0}(r) \frac{F_{y}(z, s)}{E}=0
\end{aligned}
$$

for $1 / f_{+} C \ll k_{\beta}^{2}$, with

$$
\frac{F_{y}(z, s)}{E}=\sum_{n=0}^{\infty} \frac{k_{-}}{2 f_{+}} I(z, s) \delta\left(s-\frac{z}{2}-n C\right) .
$$

By defining $W_{\perp}(z)=\sin \frac{k_{-}(z)}{2} H(z)$ with the Heaviside step function $H(z)$, we have

$$
\begin{aligned}
I(z, s) & =\int_{-\hat{z}}^{z} \sin \frac{k_{-}}{2}\left(z-z^{\prime}\right) \bar{y}\left(z^{\prime}\right) d z^{\prime} \\
& =\int_{-\infty}^{\infty} W_{\perp}\left(z-z^{\prime}\right) \bar{y}\left(z^{\prime}\right) d z^{\prime} .
\end{aligned}
$$

Note that in Eq. (15) the range of $z^{\prime}$ is actually limited to $[-\hat{z}, \hat{z}]$ via $g_{0}(r)$ in Eq. (9), due to Eqs. (11), (12) and $W_{0}(r)=\frac{k_{s}}{N_{+} \eta} g_{0}(r)$ in the following expansion in terms of uniform bunch mode $h_{m}^{|l|}(r)$

$$
\begin{aligned}
R_{l}^{ \pm}(r, s)= & W_{0}(r) \sum_{m=0}^{\infty} a_{l m}^{ \pm}(s) h_{m}^{|l|}(r) \\
h_{m}^{|l|}(r)= & \sqrt{4 \pi \frac{\left(|l|+2 m+\frac{1}{2}\right) m ! \Gamma\left(|l|+m+\frac{1}{2}\right)}{(|l|+m) ! \Gamma\left(m+\frac{1}{2}\right)}} \\
& \times\left(\frac{r}{\hat{z}}\right)^{|l|} P_{m}^{(|l|,-1 / 2)}\left(1-\frac{2 r^{2}}{\hat{z}^{2}}\right)
\end{aligned}
$$

We can see clearly from Eqs. (13)-(15) that the linear beambeam interaction acts analogously to a transverse wake function, which corresponds to the broad-band transverse impedance

$$
\begin{aligned}
W_{\perp}\left(z-z^{\prime}\right) & =\frac{-i}{2 \pi} \int_{-\infty}^{\infty}\left[Z_{\perp} c\right] e^{i k\left(z-z^{\prime}\right)} d k \\
\operatorname{Re} Z_{\perp}(k) c & =\frac{\pi}{2}\left[\delta\left(k-\frac{k_{-}}{2}\right)-\delta\left(k+\frac{k_{-}}{2}\right)\right] \\
\operatorname{Im} Z_{\perp}(k) c & =\frac{k_{-}}{4 k}\left(\frac{1}{k-k_{-} / 2}+\frac{1}{k+k_{-} / 2}\right) .
\end{aligned}
$$

Substituting Eqs. (14), (16) and (18) into Eq. (13), we finally get the equations for mode expansion coefficients $a_{\operatorname{lm} 0}^{ \pm}$ and $a_{l m}^{ \pm}$before and after a single collision:

$$
\begin{aligned}
& a_{l m}^{+}-a_{l m 0}^{+}=\sum_{l^{\prime}, m^{\prime}} M_{l m, l^{\prime} m^{\prime}}\left(a_{l^{\prime} m^{\prime} 0}^{+}+a_{l^{\prime} m^{\prime} 0}^{-}\right) \\
& a_{l m}^{-}-a_{l m 0}^{-}=-\sum_{l^{\prime}, m^{\prime}} M_{l m, l^{\prime} m^{\prime}}\left(a_{l^{\prime} m^{\prime} 0}^{+}+a_{l^{\prime} m^{\prime} 0}^{-}\right),
\end{aligned}
$$


where the coupling matrix $M_{l m, l^{\prime} m^{\prime}}$ is

$$
\begin{aligned}
& M_{l m, l^{\prime} m^{\prime}}=c_{l, l^{\prime}} \int_{-\infty}^{\infty} d k\left[Z_{\perp}(k) c\right] g_{|l| m}(k) g_{\left|l^{\prime}\right| m^{\prime}}(k), \\
& g_{|l| m}(k) \equiv C_{|l| m} J_{|l|+2 m+1 / 2}(k \hat{z}) / \sqrt{k \hat{z}} \\
& C_{|l| m}=\sqrt{\frac{(|l|+2 m+1 / 2) \Gamma(m+1 / 2) \Gamma(|l|+m+1 / 2)}{2 \pi m !(|l|+m) !}}
\end{aligned}
$$

with $c_{l, l^{\prime}} \equiv i^{l-l^{\prime}}[\operatorname{sign}(l)]^{l}\left[\operatorname{sign}\left(l^{\prime}\right)\right]^{l^{\prime}}$. Using broadband impedance and the spectrum component $g_{|l| m}(k)$ for the uniform distribution, we can show for $\bar{D}_{-}=l_{+} / f_{-}=$ $\left(\frac{l_{+}}{\sigma_{+}}\right) D_{-}, \chi=\frac{k_{-} l_{+}}{4}=\frac{\sqrt{\bar{D}}}{4}$ and $k_{\beta} \beta_{y+}^{*} \simeq 1$,

$$
\begin{gathered}
M_{l m, l^{\prime} m^{\prime}}=c_{l, l^{\prime}} \pi \xi_{+}\left[c_{o} J_{\mu}(\chi) J_{\mu^{\prime}}(\chi)\right. \\
+i c_{e}\left(\frac{(-)^{\left(\mu-\mu^{\prime}\right) / 2}}{\sin \frac{\left(\mu+\mu^{\prime}\right) \pi}{2}} J_{\max \left(\mu, \mu^{\prime}\right)}(\chi) J_{-\min \left(\mu, \mu^{\prime}\right)}(\chi)\right. \\
\left.\left.\quad-\frac{4}{\pi^{2}} \frac{\sin \frac{\left(\mu-\mu^{\prime}\right) \pi}{2}}{\mu^{2}-\mu^{\prime 2}}\right)\right]
\end{gathered}
$$

with $\mu=|l|+2 m+1 / 2, \mu^{\prime}=\left|l^{\prime}\right|+2 m^{\prime}+1 / 2, c_{o}=$ $\left[1+(-)^{|l|+\left|l^{\prime}\right|+1}\right] / 2$ and $c_{e}=\left[1+(-)^{|l|+\left|l^{\prime}\right|}\right] / 2$.

It can be shown that the full turn map from pre- $n$th collision to pre- $(n+1)$ th collision is

$$
\left(\begin{array}{c}
A^{+} \\
A^{-}
\end{array}\right)_{n+1}=\mathcal{M}\left(\begin{array}{c}
A^{+} \\
A^{-}
\end{array}\right)_{n}
$$

with

$$
\mathcal{M}=\left(\begin{array}{ll}
D_{\beta} & 0 \\
0 & D_{\beta}^{-1}
\end{array}\right)\left(\begin{array}{ll}
D_{s} & 0 \\
0 & D_{s}
\end{array}\right)\left(\begin{array}{ll}
I+M & M \\
-M & I-M
\end{array}\right)
$$

for vector element $A_{l m}^{ \pm}=a_{l m}^{ \pm}$, and matrix elemen$\mathrm{t}\left(D_{\beta}\right)_{l m, l^{\prime} m^{\prime}}=e^{-i \mu_{\beta}} \delta_{l l^{\prime}} \delta_{m m^{\prime}}$ and $\left(D_{s}\right)_{l m, l^{\prime} m^{\prime}}=$ $e^{-i l \mu_{s}} \delta_{l l^{\prime}} \delta_{m m^{\prime}}$ with $\mu_{s}=2 \pi \nu_{s}$. Here $l=0, \pm 1, \pm 2, \cdots$ and $m=0,1,2, \cdots$.

The stability of the system can be studied from the eigenvalues of $\mathcal{M}$ which are expressed as $e^{\Omega_{r}+i \Omega_{i}}$. In Fig. 1, we show $\Omega_{r}$ and $\Omega_{i} / \mu_{s}$ vs. $\Lambda \equiv D_{-} \xi_{+} / \nu_{s}$ by setting $\mu_{\beta}=0$ and varying $\xi$ from 0 to 0.01 while fixing $\bar{D}=4$ and $\nu_{s}=0.001$. The threshold of transverse mode coupling, or strong head-tail instability, occurs at $\Lambda_{t h} \simeq 6.4$. Zooming into the "stability" region $\Lambda \leq \Lambda_{t h}$ we find that there still exists small growth rate, impling synchrobetatron coupling due to localized rather than distributed beam-beam kick. The dependence of the threshold value $\Lambda_{t h}$ vs. $D_{-}$, with $\nu_{s}$ fixed to 0.001, is shown in Fig. 2. It is shown that for higher disruption $D_{-}$, the electrons oscillate through the positron bunch, and thus the system is more stable, allowing for higher threshold. We also found that for large $\Lambda$, if we fix $D_{-}$while increasing $\nu_{s}$ and $\xi_{+}$simutaneously to keep $\Lambda$ constant, the growth rates before the threshold get bigger and gradually the synchrobetatron coupling dominates over the transverse mode coupling. Further studies with strong-strong beam-beam simulation and analysis including betatron phase advance in the interaction region is currently underway.
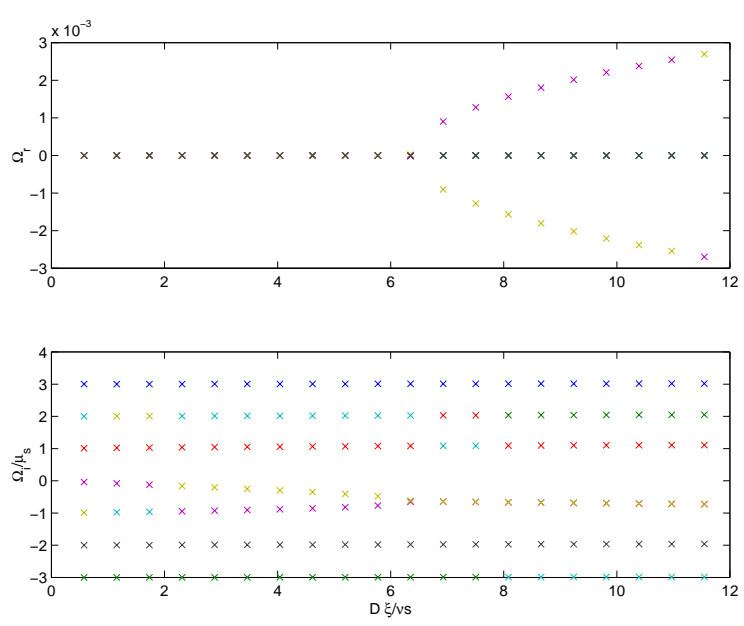

Figure 1: $\Omega_{r}$ and $\Omega_{i} / \mu_{s}$ vs. $D_{-} \xi_{+} / \nu_{s}$ by varying $\xi$ from 0 to 0.01 while fixing $\bar{D}=4$ and $\nu_{s}=0.001$.

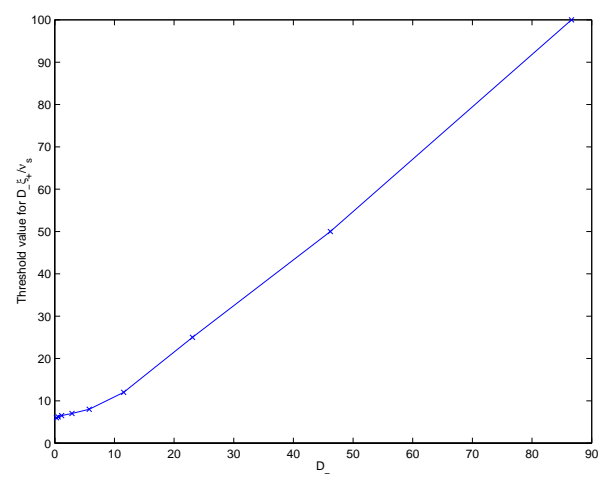

Figure 2: Threshold value of $D_{-} \xi_{+} / \nu_{s}$ at the onset of instability as a function of disruption parameter $D_{-}$, for $\nu_{s}=0.001$.

\section{REFERENCES}

[1] P. Grosse-Wiesmann, SLAC-PUB-4545, SLAC, 1988.

[2] L. Merminga and G. A. Krafft, V. A. Lebedev, Proceedings of the HEACC 2001 Conference, Japan (2001).

[3] R. Li and J. J. Bisognano, Phys. Rev. E 48, 3965 (1993).

[4] R. Li, G. Krafft and J. J. Bisognano, CEBAF-TN-93-103 (http://tnweb.jlab.org/tn/1993), 1993.

[5] E. A. Perevedentsev and A. A. Valishev, Phys. Rev. Spec. Top. 4, 024403 (2001)

[6] B. Yunn, JLab-TN-01-017 (http://tnweb.jlab.org/tn/2001), 2001.

[7] A. W. Chao, Physics of Collective Beam Instabilities in High Energy Accelerators, Wiley (1993). 